\title{
BIOMATERIALS THE BOON OF MODERN TECHNOLOGY - A GLIMPSE
}

\author{
Priyadarshini P. Maddi*
}

\section{Introduction}

'Biomaterials' are synthetic materials deployed to restore function to a body part that has been adversely affected. They are continuously or intermittently in contact with the body fluids. These materials are basically used to replace hard or soft tissues that have suffered damage due to some reason. Tissues and organs of the body, do serve for a considerable period in one's lifespan, in most people. But sometimes, they may get affected due to any of the unfortunate mishaps like fracture, infection, structural anomalies, loss of function, physical disturbances etc. During such causalities these synthetic devices loom in as 'Life- lines'. Many thanks to the relentless research of the medical bioengineers that ushered these valuable life preserving wonder devices!

Biomaterials, however do not include surgical instruments, as, though continuously exposed to body fluids they do not replace any body tissue. However, these are fabricated using special grade of stainless steel that does not react with body fluids. Biomaterials, also do not include external prosthesis like artificial limbs or devices like hearing aids, as they are not exposed to body fluids.

* Professor, Dept. of Zoology, Christ College. priya_2750@rediffmail.com 
Therefore a substance to be qualified as a biomaterial, it has to satisfy the following norms - it should

- be exposed to body fluids

- replace a body tissue that was functional in the body and has due to some reason got affected and lost its normal function needing a replacement.

- be bio-compatible i.e., should not only be protected from adverse reaction in the host, but should exhibit harmonious existence in the host body.

- be non-toxic and non-carcinogenic.

- be non-inflammatory, and non-thrombogenic.

- replace and restore function of living tissue it should possess adequate physical and mechanical properties to do so.

- be amenable to be formed or machined.

- be cost effective and readily available.

\section{Types of Biomaterials}

Biomaterials may belong to any of the four following categories viz.

- Metals

- Polymers

- Hydrogels

- Ceramics and

- Composites

\section{Metals}

Metals are widely used for load-bearing implants. They range from simple wires and screws to fracture fixation plates and artificial joints for hips, knees, shoulders, ankles etc. These are called 'total joint prosthesis'. Metallic implants are also used in maxillo-facial surgery, cardio-vascular surgery and as dental materials. The most commonly used metals are stainless steels, titanium and cobalt alloys (cobaltchromium alloys) gold, vitallium, nitinol (nickel titanium naval ordinance laboratories). Nitinol is a 'shape memory alloy', and the latest addition to the list. 


\section{Polymers}

Polymers are used in the making of facial prosthesis (prosthesis, is an artificial device used to substitute a defective or missing body part), tracheal tubes, kidney and liver parts, heart components, dentures, hip and knee joints. They are also used as medical adhesives and sealants. Egs. polyethelenes, polyurethanes, polymethylmethacrylate (PMMA).

\section{Hydrogels}

Hydrogels are biologically inert biomaterials. They include cellulose and acrylic co-polymers used in drugdelivery system, vitreous implants and wound healing dressings, artificial tendon-cartilage, artificial kidney membrane, artificial skin, maxillofacial reconstruction and vocal cord replacements.

\section{Polymers in the body}

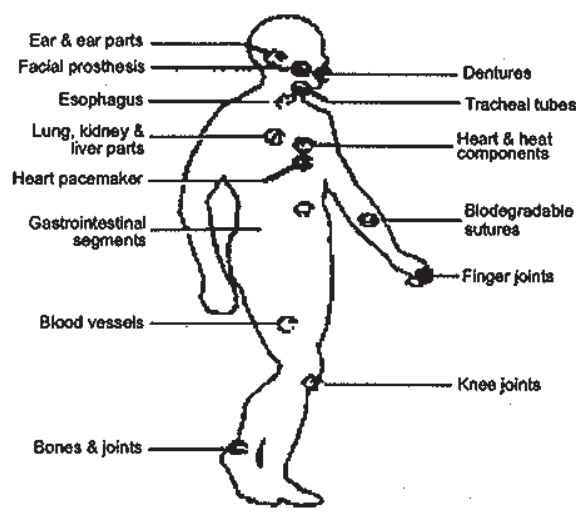

Ear \& ear parts: acrylic, polyethylene, wilicone, poly(vinyl chlaride) (PVC) Dentures: acrylic, ultrahigh motecular weight polyothylene (UHAWPE), epox Facial prosthosis: acrylic, PVC, polyurethane (PUR) Trucheal tubos: acrylic, silicore, mytor

Heart a heart components: poiyeter, silicone, PVC

Heart pacemaker: polyeltylene, acota

Lung, kidney \& Iver parti: potyester, polyailehyde, PVC

Esophagus sogmentas polyethylene. potypropyteno (PP), PVC

Blood vaseals: PVC, polyester

Blodegradable sutures: PUR

Gastrointestinal segments; silico

Bones \& Joints: acrylic, nylon, silicone, PUR, PP, UHMWPE

Kne joints: polyelthylene

\section{Ceramics}

Ceramics are widely used as restorative materials in dentistry like crowns, cements, and dentures. Due to their poor load-bearing capacity and fracture toughness they are not so popularly used in orthopaedics. However, some ceramic materials are used in joint replacement and bone repair. Egs: alumina, calcium phosphate, synthetic hydroxyapatite, pyrolytoic carbon.

\section{Composites}

Most of the composites find application in dental restorations (dental cements) and prosthetic limbs as they combine the properties of low weight/density and high strength, ideally suiting their purpose. However, for bone repair and joint replacement, though carbon-carbon and carbon reinforced polymer composites would find probable use, due to their low elastic modulus levels do not possess a combination of mechanical and biological properties desired for such applications. 


\section{Natural Biomaterials}

Several biomaterials are made from animal and plant products. The advantages of using these are:

- They are similar to living materials familiar to the body.

- Pose no problem of toxicity.

- Possess specific protein binding sites and other biochemical signals, that may help in healing and integration.

However the disadvantages that lurk in their use are:

- They pose the problem of immunogenecity

- They tend to get denatured or decomposed at temperatures below their melting points. This limits their use as implants.

The most common natural biomaterial used is collagen, finding potential use as a scaffold for neo, tissue growth, wound healing and build-up of dermal tissue for cosmetic reasons. Other natural biomaterials being considered for use include coral, chitin from insects and crustaceans, keratin from hair and cellulose from plants.

\section{Examples of Certain Biomaterials and their Applications}

\begin{tabular}{|l|l|}
\hline \multicolumn{1}{|c|}{ MATERIAL } & \multicolumn{1}{c|}{ APPLICATION } \\
\hline METALS AND ALLOYS: & \\
$316 \mathrm{~L}$ stainless steel & fracture fixation, stents, surgical instruments \\
CP-Ti (commercially pure titanium), & bone and joint replacements, fracture \\
Ti-Al-V, Ti-Mo-Zr-Fe & fixation, dental implants, pacemaker \\
encapsulation. & joint replacement, dental, heart valves \\
Co-Cr-Mo, Cr-Ni-Cr-Mo & bone plates, stents, orthodontic wires \\
Ni-Ti & dental restorations \\
Gold alloys & \\
\hline
\end{tabular}




\begin{tabular}{|c|c|}
\hline CERAMICS AND GLASSES: & \\
\hline Alumina & joint replacements, dental implants \\
\hline Zirconia & joint replacement \\
\hline Bioactive glasses & bone replacement \\
\hline Porcelain & dental restorations \\
\hline Carbons & $\begin{array}{l}\text { heart valves, percutaneous device } \\
\text { dental implants }\end{array}$ \\
\hline \multicolumn{2}{|l|}{ POLYMERS: } \\
\hline Polyethylene & joint replacement \\
\hline Polypropylene & sutures \\
\hline Polyethylene terephthalates (PET) & sutures, vascular prosthesis \\
\hline Polyamides & sutures \\
\hline Polytetrafluoroethylenes (PTFE) & $\begin{array}{l}\text { soft tissue augmentation vascular } \\
\text { prosthesis }\end{array}$ \\
\hline Polyesters & vascular prosthesis, drug delivery systems \\
\hline Polyurethanes & blood contacting devices \\
\hline Polyvinyl chlorides (PVC) & tubing \\
\hline Polymethylmethacrylate (PMMA) & $\begin{array}{l}\text { dental restorations, intra occular lenses, } \\
\text { joint replacements (bone cements) }\end{array}$ \\
\hline Silicones & soff tissue replacement, ophthalmology \\
\hline Hydrogels & ophthalmology, drug-delivery systems \\
\hline \multicolumn{2}{|l|}{ COMPOSITES : } \\
\hline BIS-GMA-quartz/silica filler & dental restorations \\
\hline PMMA-glass fillers & dental restorations (dental cements) \\
\hline
\end{tabular}

\section{Certain Applications of Biomaterials}

\section{In Orthopaedics}

Several orthopaedic implant devices are fabricated with biomaterials. Free movements of synovial joints like hip, knee, shoulder, ankle and elbow may be affected due to both osteoarthritis and rheumatoid arthritis. The pain in such joints, 
particularly weight-bearing joints such as hip and knee, is considerable. With the judicious administration of anesthesia, antiseptics and antibiotics, all of these can be successfully replaced with prosthesis relieving pain and restoring mobility to scores of sufferers. A natural synovial joint consists of layers of bearing material (articular cartilage) mounted on hard bones that form the skeletal framework. The synovial fluid acts as the lubricant.

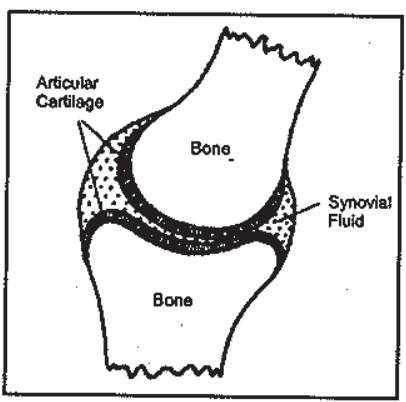

In an artificial joint lubrication is supplied by low friction polymeric bearing materials. A biomaterial used for joints must necessarily possess certain additional features like:

- excellent resistance to biological degradation in polymers and corrosion resistance for metals.

- strength to bear load.

- minimal bone resorption.

- high wear resistance to minimize wear-debris generation.

\section{Total Hip Replacement}

Total hip replacement is a major land mark event of the $20^{\text {th }}$ century. This was done with the creation of a new joint through a procedure called 'arthoplasty' where a degenerate joint was surgically treated. It was done to relieve pain and restore movement. Femur (thigh bone) and the pelvis (hip bone) were replaced, and the mating pelvis (hip bone) replaced or resurfaced.

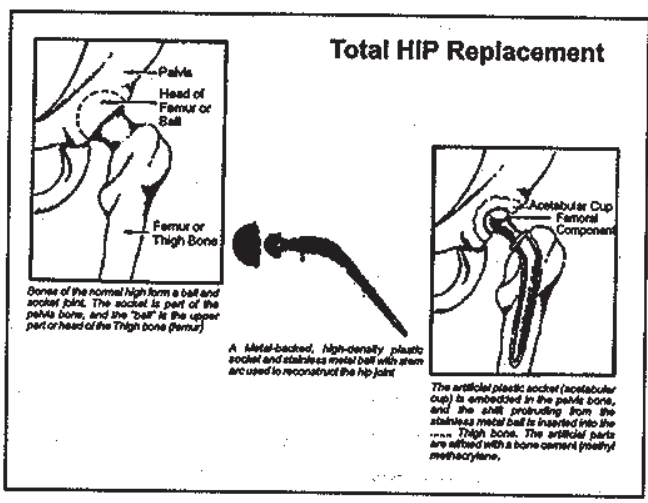

A typical hip prosthesis consists of the femoral stem, a femoral ball and a polymeric socket (cup) made of UHMWPE (ultra high molecular weight polyethylenes) with or without metallic backing. The femur stem, ball and socket are usually made of $\mathrm{Co}$ $\mathrm{Cr}-\mathrm{Mo} / \mathrm{Co}-\mathrm{Ni}$-Cr-Mo / titanium alloys. 


\section{Total Knee Replacements}

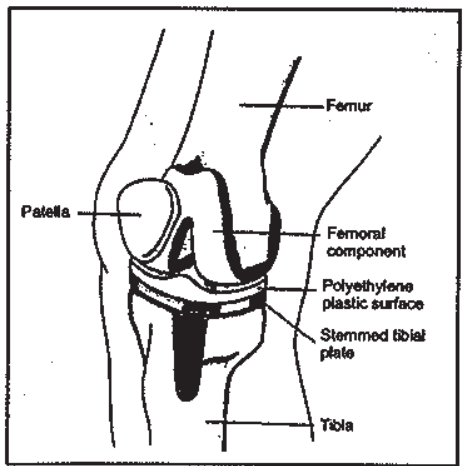

This is also called total knee arthoplasty (TKA). In this surgical procedure diseased cartilage surfaces of lower femur (thigh bone), tibia (shin bone) and patella (knee cap) are replaced by prosthesis made of alloys viz; titanium alloys (Ti$6 \mathrm{Al}-4 \mathrm{~V}$ ) and cobalt - chromium alloys and polymeric materials made of UHMWPE anchored to a metal plate about half a kilogram. The three bones replaceable employing TKA are: the lower ends (condyles) of the thigh bone, the top surface of the shin bone and back surface of the kneecap. The designing of the components is such that, metal always articulates against a low-friction plastic, that facilitates smooth movement causing minimal wear.

The metal femoral component, curves around the end of the thigh bone with an interior groove for the smooth up and down movement of the kneecap against the bone, as the knee bends and straightens.

The tibial component, is a flat metal platform with a polymeric cushion.

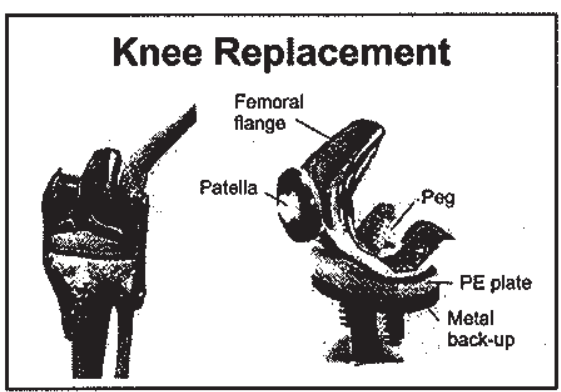

The patellar component, is a dome-shaped piece of polyethelene that duplicates the shape of the kneecap anchored to a flat metal plate.

\section{In Ophthalmics}

The tissues of the eye may suffer from several abnormalities causing reduced vision and even blindness. Intraoccular lenses deployed to replace the cloudy lenses developed during cataract, and contact lenses, restore normal vision. Both of these are in constant contact with the tissues of the eye. These are fabricated using biomaterials that are synthetic polymers, Polymethylmethacrylate (very transparent), and hydrogels. Infact hydrogels found earliest application in the making of contact lenses due to good mechanical stability, favourable refractive index and high oxygen permeability exhibited by them. 


\section{Dental Application}

Inside the mouth both the tooth and the gums are prone to bacterial infection. Tooth loss is caused due to:

- Dental cavities (carries)

- Demineralisation/dissolution of teeth

- Plaque formation

Entire or their segments, can be replaced by a variety of biomaterials like ceramics, porcelein, PMMA etc. Dental restorations are done using gold alloys, $\mathrm{Hg}-\mathrm{Ag}-\mathrm{Sn}$ amalgams, carbons, BIS-GMA quartz silica fillers and PMMA-glass fillers.

\section{Wound Healing}

The earliest use of implantable biomaterials was in suturing wounds. Ancient Egyptians in $2000 \mathrm{BC}$ used linen for the purpose. The synthetic suture biomaterials include polymers and metals like special stainless steel, tantalum and hydrogels. The resorbables used in sutures are polyglycolic acid polyacetic acid and polyesters. Fracture fixation devices include bone plates, screws, nails, rods, wires etc. These are made of stainless steel. However uses of some non-metallic materials like carboncarbon composite bony plates are under investigation.

\section{Drug-delivery System}

For sustained and controlled release of drugs, implantable devices are made as drug reservoirs and fixed onto certain specific places in the body. Hydrogels and resorbables are used for the purpose. Egs. MEMS; in the release of insulin in diabetes.

\section{Cardiovascular Applications}

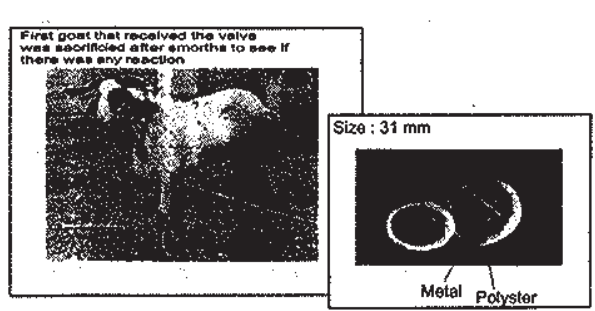

Heart valves and arteries can be substituted using biomaterials. Structural changes can affect heart valves, hindering their complete opening or closing. Biomaterials used are ceramics, metals and polymers. Eg. coronary arteries and vessels of the lower limbs blocked due 
to fatty deposits, called atherosclerosis, replaced by artificial arteries made of polymers are called vascular prosthesis.

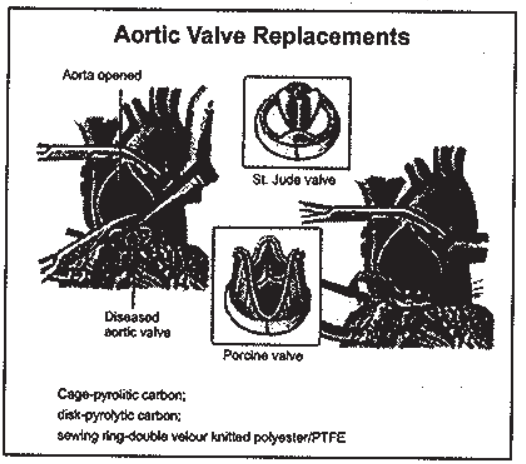

\section{Cardiac Pacemakers}

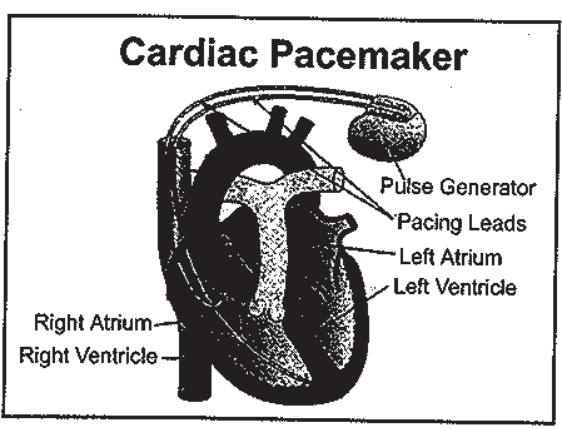

by is unable to regulate the functioning of the heart it calls for immediate replacement with an artificial device that can restore the affected function. This is done implanting a cardiac pacemaker.

The cardiac pacemaker consists of a pulse generator, 1 or 2 electrodes and 1 or 2 pacing leads connecting the pacemaker to the heart. The system is designed to send precisely timed electrical signals to induce muscle contraction creating an impulse very similar to that generated by a naturally working heart. The artificially fabricated pacemaker is implanted between the skin and pectoral muscle. Sealed lithium-iodine battery provides the electrical energy. This battery has extended the life of some pacemakers over ten years in comparison with the mercury-zinc batteries used before 1975. The circuit not only converts the electrical energy into electrical signals, but also controls the timing of the electrical signals delivered to the heart.. 
The components of the pacemaker include, connector block, pulse generator, pacing lead with a connector pin, a lead body, and electrodes.

The connector block attaches the pacemaker to the lead. It is located on top of the pacemaker and is made of polyurethanes. Earlier they were made of glass.

The pulse generator is encased in ASTM grade 1 titanium. Earlier ceramics and epoxy resins with silicone rubber were used for the same purpose. The advantage of titanium is that it provides protection against external electromagnetic interference.

The pacing lead transmits electrical signals from the pacemaker to the heart, and information about the heart activity from the heart to the pacemaker. One or two leads may be used. One end of the lead is attached to the connector block and the other inserted through a vein and placed in the right ventricle or right atrium.

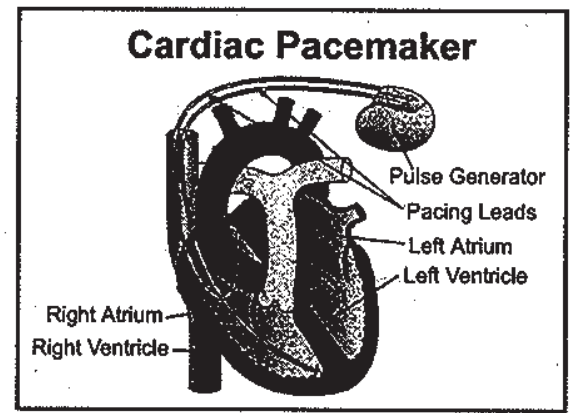

A lead is an insulated wire consisting of:

- Connector pin, inserted into the connector block.

- Head body, an insulated metal wire, carrying electrical signal from the pacemaker to the heart.

- Fixation mechanism, that helps to anchor the tip of the lead containing the electrodes at the appropriate place in the heart. It is made of a Ni-Co alloy with a silver core helix or electrically active platinum-iridium helix.

- Electrodes made of platinum, titanium, stainless steel, silver or cobalt alloys. Titanium forms a non-conducting oxide layer at the surface. It prevents exchange of charge carriers across boundaries making it inert. Some of its other properties are elasticity, high resistance to corrosion and high durability. The electrode tip promotes the development of fibrotic tissue growth physically stabilizing the tissue interface.

The leads are made steroid eluting to suppress inflammatory responses of the heart wall. The steroid used is dexamethasone sodium phosphate. The requirements of a lead are that it must be - 
- able to withstand flexing induced by cardiac contractions in the warm and corrosive environment of the body through its inert, non-toxic, durable nature.

- a good electrical conductor.

- strong. Replacement of polyethylenes used earlier by polyurethanes and silicone rubber tubing helped to achieve this feature. Polyurethanes have made the leads thinner and more flexible. The coefficient of friction is less when wet. As again, polyurethanes are degradable by metal-ion induced oxidation the use of silicone rubber tubing is more advantageous.

\section{Shape Memory Alloys}

Shape memory alloys, SMA for short, are new generation material in biomedical applications. These are metals exhibiting shape memory properties. The 'shape memory effect' was discovered in 1932 in silver-cadmium alloy. Materials possessing this property return to their original shape after having suffered some form of deformation after they are heated to temperatures above their transformational phase. SMA can recover from small and large deformations, within recoverable ranges. The process of deformation can be repeated a million times. Although commercially avaitable shape memory alloys include, nickel-titanium, copper-zincaluminium, copper-alumium-nickel etc only nickel-titanium ( $\mathrm{Ni}-\mathrm{Ti}$ alloy) is suitable for biomedical applications.

The applications of shape memory alloys include:

- Stent: Is a device to treat a coronary disease. The Ni-Ti alloy is deformed during insertion into the blocked artery, and upon reaching the body temperature expands and opens it increasing blood flow. This super elastic medical device was developed in the 1980s. It was named after Dr. C.T. Stent who had developed it as a dental device

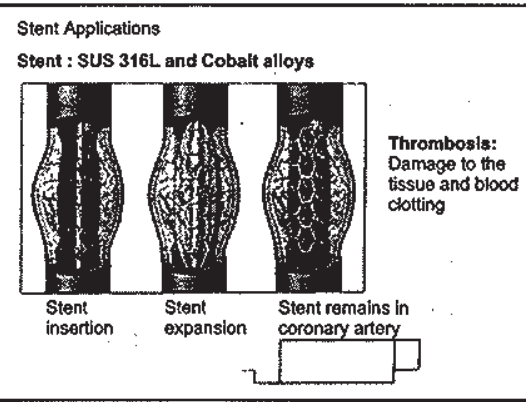
much earlier

- Venacava filter: Is a device used to trap blood clots. The SMA is inserted as a small cylinder. It reverts to an umbrella shaped filter to trap small blood clots checking their reach to other parts of the body preventing their detrimental effects. 
- Dental and orthodontic arch wires: These work like a spring. They apply a continuous and gentle force correcting misaligned teeth as opposed to the periodic and uncomfortable tightening required by stainless steel wires.

All biomaterials have to be tested before implantation according to the FDA regulation. Adverse reactions that may be elicited by the host are rapid dialation of capillaries and release of degenerative enzymes, the lysozymes, by macrophages to digest the foreign material. Other factors to be considered before implanting a biomaterial are that, species tests done in other animals need not necessarily hold good for humans. The age and health status of the person is very important. The implantation should be properly done in such a manner that the tissue damage is minimal and rid of any contamination.

So, while biomaterials are indeed a boon their use has to be extremely sane!

\section{Acknowledgement}

The information contained in this article was gathered during a seminar on "Biomaterials and their applications in medicine" conducted at M.S.Ramaiah School of Advanced Studies.

\section{References}

1. M. Long and H.J. Rack, Titanium alloys in Total Joint Replacement - A Materials Science Perspective, Biomaterials, Vol. 19, 19983, p. 1621 - 1639.

2. D. Williams, An Introduction to Medical and Dental Materials, Concise Encyclopedia of Medical \& Dental Materials, D. Williams, Ed., Pergamon Press, 1990, p. xvii - xx.

3. P. Didisheim and J.T. Watson, Cardiovascular Applications, Biomaterials Science: An Introduction to Materials in Medicine, B.D. Ratner, A.S. Hoffman, F.J. Schoen and J.E. Lemons, Ed., Academic Press, 1996, p. $283-297$.

4. G. Heinke and P. Griss, Ceramic implant materials, Med. Biol. Eng. Comput., Vol. 18, 1980 , p. $503-510$.

5. A. J. Klein, Bio materials give new life, Adre. Mater. Process, May 1986, p. 18 - 22.

6. D. C. Mears, Metals in Medicine and Surgery, Int. Met. Rev., Vol. 22, June 1977, p. $119-155$.

7. D. E. Niesz and V.J. Tennery, Ceramics for prosthetic applications - Orthopaedic, dental and Cardiovascular, Metals and Ceramics Information Centre Report July 1974.

8. D. F. Williams, Corrosion of implant Materials, Ann Rev. Mater. Sci., Vol 22, 1987, p. $3241-3445$.

9. K, R St. John, Bio- compatibility Testing for Medical Implant Materials, ASTM Stand. News, Morch 1994, p. 46. 49. 